\title{
Proyecto de intervención socioeducativa: contexto social y educativo, caso Escuela Primaria Hermenegildo Galeana
}

\author{
E. N. Hiram Fernando Arizmendi \\ arizmendihiram.aritmetica@gmail.com \\ Dr. en Ed. Carlos Israel Hannz Sámano \\ hannzcarlos@gmail.com
}

Centenaria y Benemérita Escuela Normal para Profesores

Recibido: 05 de febrero del 2020

Aceptado: 05 de marzo del 2020

\section{Resumen}

El Proyecto de Intervención Socioeducativa (PISE), tiene el propósito de conocer por medio de un diagnóstico y la observación, la situación social y educativa de una institución, la finalidad es realizar una intervención educativa para fortalecer el área o áreas detectadas. El PISE, se realizó en la Escuela Primaria Hermenegildo Galeana de la ciudad de Toluca Estado de México, del 03 al 14 de junio de 2019, a partir de los datos obtenidos se identificaron algunas problemáticas de las cuales la principal fue la convivencia escolar pacífica a nivel institucional, por grado y por grupo con el fin de promoverla. La metodología que se utilizó para la implementación del proyecto fue la propuesta por Ander Egg (1998). Al proyecto se le denominó "Convivir para aprender en el aula"

Palabras clave: Proyecto de intervención socioeducativa, convivencia escolar pacífica.

\begin{abstract}
The Socio-Educational Intervention Project (PISE), has the purpose of knowing, by means of a diagnosis and observation, the social and educational situation of an institution. The aim is to carry out an educational intervention to strengthen the area or areas detected. The PISE was held at Hermenegildo Galeana Elementary School in Toluca, State of Mexico, from June 3 to 14, 2019. From the data obtained, some problems were identified, the main one being peaceful school coexistence at institutional level, by grade and by group in order to promote it. The methodology used for the implementation of the project was proposed by Ander Egg (1998). The project was called "Living together to learn in the classroom"
\end{abstract}


Keywords: Socio-educational intervention project, peaceful school coexistence

\section{Presentación}

El siglo XXI, se caracteriza por los avances tecnocientíficos y trasformaciones sociales en el orbe global; por ello, en el sistema educativo se establecen acciones específicas para atender los acontecimientos socioeducativos ocurridos, de lo cual se ha generado movilidad social en los paralelismos económico y educativo principalmente.

El desarrollo y movilidad social desde las Instituciones educativas, se han estructurado bajo estrategias y programas para fortalecer y crear mejores espacios de convivencia escolar, ante ello se inserta el Proyecto de Intervención Socioeducativa (PISE), el cual se desarrolla como estrategia de intervención educativa desde un diagnóstico situacional del contexto educativo y social de la Escuela Primaria Hermenegildo Galeana de la ciudad de Toluca Estado de México, ubicada en Av. 16 de septiembre, en la localidad de Santa Ana Tlapaltitlán.

El PISE, se realizó del 03 al 14 de junio de 2019 bajo la conducción de docentes y la planificación y ejecución de estudiantes del sexto semestre de la Licenciatura en Educación Primaria de la Centenaria y Benemérita Escuela Normal para Profesores, así como diversos recursos humanos, materiales y financieros; las actividades planificadas del proyecto de intervención se encaminaron para responder a las necesidades del contexto escolar, vinculando a los agentes educativos: a) directivos; b) maestros; c) padres de familia; d) alumnos.

Para desarrollar el proyecto de intervención se identificó con anterioridad una problemática desde un diagnóstico situacional, realizado durante la primera jornada de práctica educativa mediante diversos instrumentos de recolección de datos como la guía de observación $\mathrm{y}$ un cuestionario de preguntas abiertas y cerradas.

La información arrojó datos, los cuales fueron procesados, analizados e interpretados y permitieron identificar con claridad la problemática, misma que giró en torno a la convivencia escolar, este hecho tiene relación con el aprendizaje, aplicación y reflexión en torno a los valores y principios que caracterizan a un sujeto. 
El PISE se denominó "Convivir para enseñar en el aula", el cual tuvo como principal objetivo promover estrategias didácticas que fortalecieran una convivencia escolar pacífica a través de diversas acciones a nivel institucional, por grado y por grupo, además se implementaron dos estrategias incorporadas al proyecto general convivir es reír y compartir es vivir bajo una intervención didáctica, la cual consistió en una planeación estratégica y transdisciplinar aplicada y enfocada a fortalecer la convivencia escolar pacífica.

El PISE, se sintetiza en la siguiente estructura: preparación, planificación, desarrollo, implementación, evaluación y resultados, referencias y anexos de las actividades realizadas.

\section{Etapa 1: Preparación del PISE}

Las actividades institucionales por grado y por grupo, fueron diseñadas con el fin de: transformar la realidad de la escuela y crear una convivencia escolar más armónica. Preparar una planificación didáctica la cual permita trabajar la sana convivencia en los centros escolares, y conlleva a seleccionar cuidadosamente cada una de las actividades para desarrollar adecuadamente una convivencia pacífica.

El análisis para llevar a cabo las actividades se sustenta desde un diagnóstico situacional, donde el contexto social y escolar fue determinante para integrar acciones encaminadas a fomentar la convivencia. La preparación del diagnóstico del PISE, fue necesario revisar una investigación sobre el contexto social donde está inmerso el centro educativo, además la búsqueda en periódicos, revistas y otras fuentes de información para conocer las principales características del lugar (fuentes de empleo, nivel socioeconómico, centros recreativos, culturales, y diversas problemáticas como la inseguridad, inasistencia sanitaria en calles, entre otros).

En la jornada de observación del centro educativo, se identifican problemáticas de violencia, contaminación ambiental y ausencia de convivencia escolar pacífica entre los alumnos; el análisis de la información se realizó por medio de un listado de datos, jerarquizándolos y codificando el nivel de importancia y relevancia desde la comunidad a la escuela, por ello se abordó el proyecto "Convivir para enseñar en el aula", desde un enfoque ético reflexivo y aplicativo de los valores universales. 
El proyecto de intervención socioeducativa, se desarrolla desde la estrategia pedagógica denominada escuela activa, su fundamento se sustenta en una filosofía pragmática, es decir aprender haciendo, a fin de obtener un aprendizaje más significativo a través de actividades de experimentación e interacción con el ambiente escolar (sujetos y objetos).

La preparación del PISE, consistió en integrar una serie de aspectos pedagógicos, teóricos, estratégicos y pasos desde su preparación hasta su evaluación, según Ander Egg (1998), "Ningún proyecto puede realizarse sin una sucesión de quehaceres y aconteceres que tienen el propósito de transformar ciertos insumos en los resultados previstos (productos) dentro de un período de tiempo determinado" (p.19).

La investigación para el desarrollo del PISE, se integró bajo los pasos según autores como Ezequiel Ander Egg y María José Aguilar Idáñez (Tabla 1).

\begin{tabular}{|l|l|l|}
\hline 1. ¿Qué? & Se quiere hacer & Naturaleza del proyecto \\
\hline 2. ¿Por qué? & Se quiere hacer & Origen y fundamentación \\
\hline 3. ¿Para qué? & Se quiere hacer & Objetivos, propósitos \\
\hline 4. ¿Cuánto? & Se quiere hacer & Metas \\
\hline 5. ¿Dónde? & Se quiere hacer & $\begin{array}{l}\text { Localización física } \\
\text { (ubicación en el espacio) }\end{array}$ \\
\hline 6. ¿Cómo? & Se va hacer & $\begin{array}{l}\text { Actividades y tareas. } \\
\text { Metodología }\end{array}$ \\
\hline 7. ¿Cuándo? & Se va hacer & $\begin{array}{l}\text { Calendarización o cronograma } \\
\text { (ubicación en el espacio) }\end{array}$ \\
\hline 8. ¿A quiénes? & Va dirigido & Destinatario o beneficiarios \\
\hline 9. ¿Quiénes? & Lo van hacer & Recursos humanos \\
\hline 10. ¿Con qué? & $\begin{array}{l}\text { Se va hacer } \\
\text { Se va a costear }\end{array}$ & $\begin{array}{l}\text { Recursos materiales } \\
\text { Recursos financieros }\end{array}$ \\
\hline
\end{tabular}

Tabla 1. Diez preguntas para diseñar un proyecto propuesto por Ander-Egg y Aguilar (1998).

\section{Etapa 2: Planificación de la jornada de práctica}

Los cursos que intervienen del plan de estudios de Educación Primaria 2012 son: 


\subsection{Filosofía de la educación}

Este curso permitió conocer la dimensión filosófica del PISE y del proyecto didáctico, a través de la enseñanza de los métodos globalizados, partiendo de la educación progresista y liberadora; con ello proveer un argumento y sustento filosófico a la implementación del método de proyectos realizado en la escuela de práctica, tomando como principales referentes a John Dewey y William Killpatrick. A partir de la base filosófica del curso, surgen las interrogantes ¿La importancia de estimular el pensamiento filosófico en niños y niñas?, ¿Estimular el pensamiento crítico y reflexivo en niños y niñas?

\section{2. Diagnóstico e intervención socioeducativa}

Este curso aportó la realización del diagnóstico situacional para la identificación del problema sociocultural en la comunidad donde se localiza la escuela primaria, la integración teórica fue desde Ander Egg (1998), Trilla (2004), Risco (2010), entre otros, los cuáles fueron fundamentales para sustentar la intervención socioeducativa en la escuela primaria permitiendo argumentarla; además aprender el cómo se estructura y las características de cada etapa del proyecto de intervención socioeducativa y el su respectivo informe.

\subsection{Formación Cívica y Ética}

El curso se integró al proyecto de intervención gracias a su objeto de estudio desde la ética y la práctica de valores; el curso posibilitó desarrollar competencias para la vida y obtener una base epistemológica fundamentada en valores y civismo que influyen directamente en la convivencia escolar pacífica y en la educación democrática, desde autores como Adela Cortina, Emmanuel Kant, Ortega y Gasset.

\subsection{Educación Geográfica}

El curso permitió realizar actividades relacionadas al tema de convivencia, desde un enfoque geográfico, identificando los componentes y características naturales, sociales, culturales y lingüísticos, estos componentes, se aprecian en la vida diaria de toda sociedad; además se tomó el día mundial del medio ambiente del 5 de junio del presente año, como actividad inclusiva y sensitiva. 
2.5. Educación artística (artes visuales y teatro)

Este curso se enfocó a desarrollar actividades institucionales por medio del proyecto teatral con sus respectivos lenguajes artísticos con la puesta en escena de una obra de teatro el día 14 de junio de 2019 en dos horarios: 08:00 a 09:15 y 09:30 a 10:45 de la mañana; la obra se elaboró de manera grupal con base en la problemática detectada "convivencia escolar"

\subsection{Producción de textos académicos}

Este curso optativo permitió realizar previamente los aspectos que se requieren para escribir un texto, de lo cual se orilló a dar sugerencias a diferentes escritos y a la planeación didáctica, aunado a esto, se dieron elementos y sintácticos necesarios para la realización del presente proyecto de intervención socioeducativa.

\subsection{Inglés B1}

Este curso permitió la realización de actividades encaminadas de grupo para promover el tema de convivencia con señalamientos en inglés en: baños, pasillos, salones y otros.

\subsection{Proyecto de intervención socioeducativa}

Este curso, fue la base para conocer la problemática vinculando el curso Diagnóstico e intervención socioeducativa y desarrollar y articular las actividades a nivel institucional, por grado y grupo sobre las cuales se debía intervenir. El curso permitió realizar en las jornadas de observación y de práctica competencias y conocimientos adquiridos en los semestres anteriores de formación inicial. Al mismo tiempo, dio la base para la realización del proyecto didáctico con base en la Guía de Proyectos didácticos de la SEP (2013) y los planes y programas de estudios de la SEP (2011).

\section{Etapa 3: Desarrollo del PISE}

La metodología del proyecto una vez definida, se planearon estrategias y actividades a implementar a nivel institucional coordinadas con actividades de grado y grupales. 
El trabajo por proyectos didácticos es una propuesta de enseñanza que permite el logro de propósitos educativos, por medio de un conjunto de acciones, interacciones y recursos planeados y orientados a la resolución de un problema o situación concreta y a la elaboración de una producción tangible o intangible (SEP, 2011, p.28).

Primeramente, se optó por realizar una conferencia para integrar a los padres de familia sobre la importancia de fomentar valores en el hogar, con el propósito de sensibilizarlos. Después se ejecutaron actividades institucionales, por grado y grupo enfocadas a la convivencia escolar pacífica.

Por último y para culminar las actividades institucionales, se realizó la obra de teatro "El mago de Oz" la cual se sustenta bajo los valores como el respeto y la honestidad, además la importancia de la resiliencia como factor de adaptación a situaciones adversas, la obra teatral surge desde el curso de Educación artística, el cual se perfilo para integrar la interdisciplinariedad de la asignatura y se desarrolló desde las fases de un proyecto, desde el referente del libro de Enseñanza Situada de Díaz Barriga (2006) quien plantea las concepciones actuales sobre los proyectos refiriéndose; y la metodología empleada por Ander Egg (1998), Selma Wasserman (1964) y William Kilpatrick (1921). (Tabla 2)

\begin{tabular}{|l|l|}
\hline Propósito & Elección del tema con base en las necesidades de los alumnos. \\
\hline Planificación & $\begin{array}{l}\text { Organiza los espacios, tiempos y recursos; se diseñan las actividades que } \\
\text { se van a desarrollar en el programa. }\end{array}$ \\
\hline Ejecución & Se implementa el proyecto. \\
\hline Evaluación & Se evalúa el proyecto en función del alumnado, el docente y el proyecto. \\
\hline
\end{tabular}

Tabla 2. Diseño de modelo propio de las fases del proyecto a partir de la clasificación de Kilpatrick (1921).

Durkheim hace referencia que la educación debe formar a los alumnos para una vida social, dicha educación influye en la moral que exigirá el niño en su contexto social, por lo que, es importante transmitir la sana convivencia, a través de la educación para que la sociedad transfiera una moral adecuada a las futuras generaciones. En ese sentido, es importante trabajar los valores para la convivencia en la educación básica; por consiguiente, se planeó en el PISE "Convivir en las aulas" y "Convivir es reír, compartir es vivir" para favorecer que los alumnos convivan en el aula y la escuela. 


\section{Etapa 4: Implementación del PISE}

Las actividades del PISE fueron dirigidas a la temática central de convivencia, mismas que fueron diseñadas con fundamentos de la pedagogía progresista, es decir, aprender haciendo, Dewey (1959) señala que "Los factores fundamentales de la educación son dos: por un lado, un ser no desarrollado, no maduro y, por el otro, ciertos fines sociales, ideas, valores que se manejan a través de la experiencia madura del adulto" (cita en Dorantes, 2007, p.3)

Las actividades implementadas por los docentes en formación para la jornada de práctica fueron articuladas desde el trabajo colaborativo. La SEP (2011) señala "Que el trabajo colaborativo alude a estudiantes y maestros, y orienta las acciones para el descubrimiento, la búsqueda de soluciones, coincidencias y diferencias, con el propósito de construir aprendizajes en colectivo" (p. 28).

\subsection{Actividades instituciones:}

Primera) Se realizó la conferencia a padres de familia denominada "La Participación de la Familia en el Manejo de las Emociones" (ver anexo 1) el día 4 de junio de 2019. La conferencia la dictó el Dr. Carlos Israel Hannz Sámano, en ella asistieron padres de familia y fue en dos horarios a las 8:00 de la mañana y a las 12:00 del mediodía. Debido a eso se planteó necesario el desarrollar una conferencia donde los padres de familia reconocieran la importancia de fomentar un manejo de emociones a través del recomendaciones y acciones específicas para convivir pacíficamente bajo el binomio escuela-hogar. "La Educación Socioemocional es un proceso de aprendizaje a través del cual los niños y los adolescentes trabajan e integran en su vida los conceptos, valores, actitudes y habilidades que les permiten comprender y manejar sus emociones, y construir" (SEP, 2017, p. 420).

Segunda) Se llevó a cabo la actividad denominada "Pintar juegos de patio" (Figura 1), la cual, consistió en dar color a figuras y siluetas dibujadas en los tres patios de la escuela, en esta actividad, participaron todos los estudiantes del sexto semestre.

Los juegos de patio tienen un enfoque lúdico y recreativo, pasan a formar parte de un espacio en donde los alumnos se sientan contentos, desarrollen habilidades sociales y motrices de una forma relajada, las experiencias que vivan serán afines, el juego y a la diversión (SEP, 2013). 


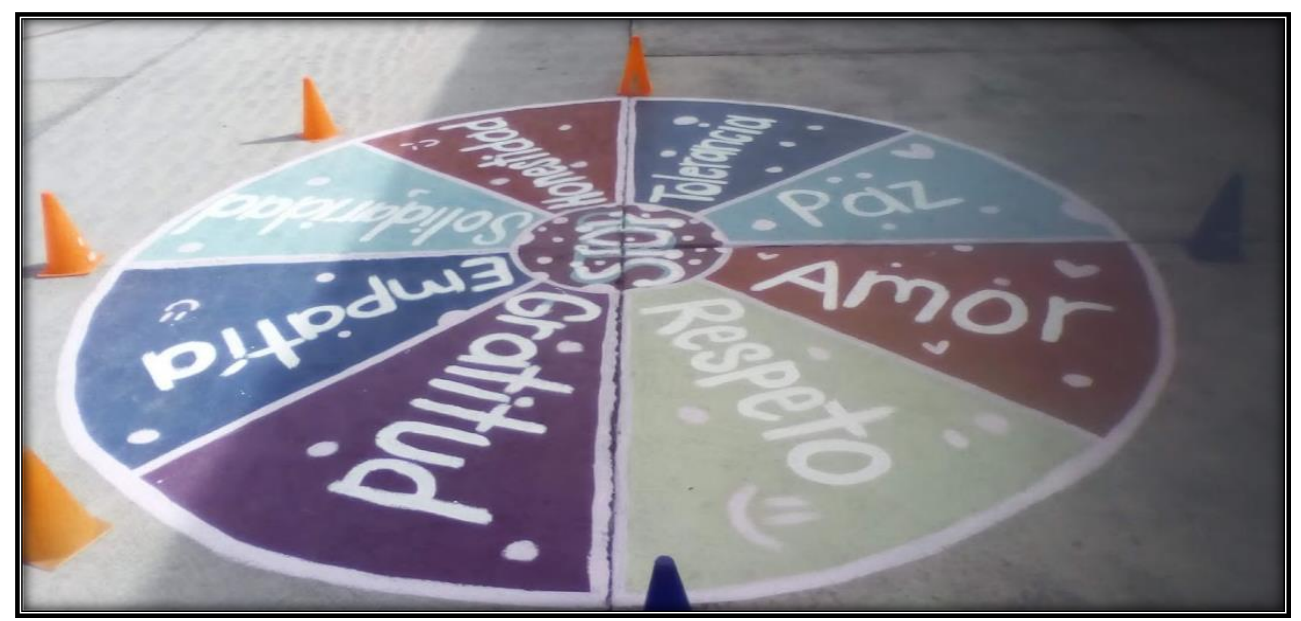

Figura 1. Pintar juegos de patio, actividad lúdica denominada Stop pintado en el patio central de ceremonias de la Escuela Primaria "Hermenegildo Galeana"

En cuanto a la convivencia escolar, desde una mirada de transformación es vista como la formación en valores sociales y humanos, así como la generación de acciones que vinculan la convivencia escolar con el ethos (costumbre y conducta) escolar, generando identidad de estudiantes, promoviendo acciones que trasciendan en el aula y previniendo fenómenos como la violencia (López, 2014). De aquí, la decisión teórica de implementar juegos como actividad fuera del aula y con un enfoque lúdico y recreativo.

Tercera) se desarrolló la obra de teatro el mago de Oz (Figura 2), la cual tiene el propósito de favorecer los valores; la primera función dio inicio a las 8:10 de la mañana el día 14 de junio de 2019, el público presente fueron alumnos de los grados de segundo, cuarto y sexto. La segunda función comenzó a las 09:30 y el público asistente fuero los grados de primero, tercero y quinto.

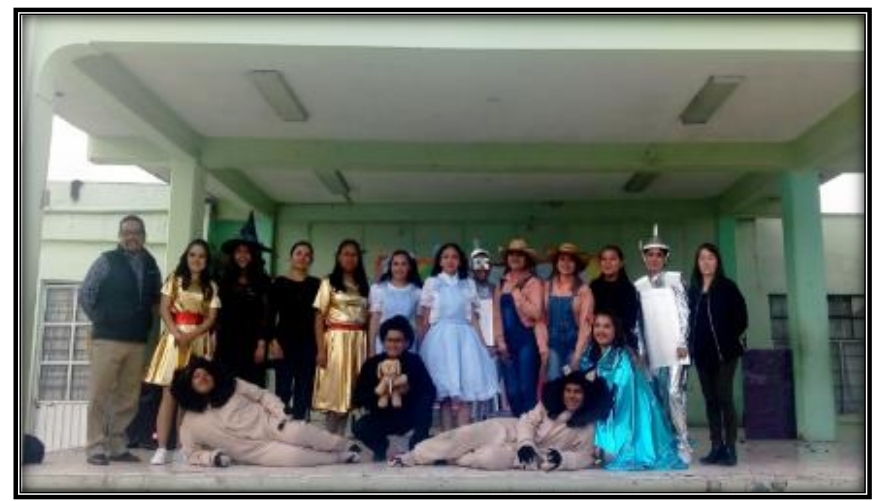

Figura 2. Presentación de estudiantes de sexto semestre de la Licenciatura en Educación Primaria de la obra de teatro "El mago de Oz" 


\subsection{Actividades por grado}

El cine fue una actividad con el propósito para identificar valores y pensamiento crítico en la vida diaria de alumnos de $6^{\circ}$; al final del set se permitió interactuar entre alumnos de diferentes salones para intercambiar aspectos de la película y fomentar habilidades sociales a través de la convivencia escolar pacífica. El rally de la convivencia se realizó en un horario de 09:00 a 10:30 hrs. de la mañana, la actividad persiguió como propósito generar una convivencia sana entre todos los alumnos del $6^{\circ}$ de una forma recreativa y lúdica, fuera del aula de clases en equipos de trabajo bajo cuatro estaciones:

- Primera, correspondía a un reto de fuerza física y desarrollo de habilidades motrices desde los juegos cooperativos para la paz.

- Segunda, desafío de razonamiento lógico-matemático.

- Tercera, un reto cooperativo y cuestionamientos sobre la convivencia.

- Cuarta, realizar un reto de destreza motriz en la cuál por equipos competían para ganar en un torneo de bolos.

La feria de la convivencia fue un espacio donde los alumnos mostraban las producciones realizadas, el propósito de la actividad fue que los alumnos hicieran exposiciones en soneto sobre la convivencia, y explicaron ¿qué era un soneto ¿y? ¿cuáles eran sus características?.

\subsection{Actividades por grupo}

Las actividades por grupo (Figura 3) se determinaron según las características y estilos de aprendizaje de los alumnos para apoyar las actividades del PISE, se consideró el contexto de la comunidad y el escolar; así como a los alumnos con Barreras para el Aprendizaje y la Participación (BAP) para la implementación de las actividades grupales. Esto se realizó, sin perder de vista los aprendizajes esperados y los contenidos de las diferentes asignaturas. A continuación, se muestran las actividades que se implementaron para el PISE. 


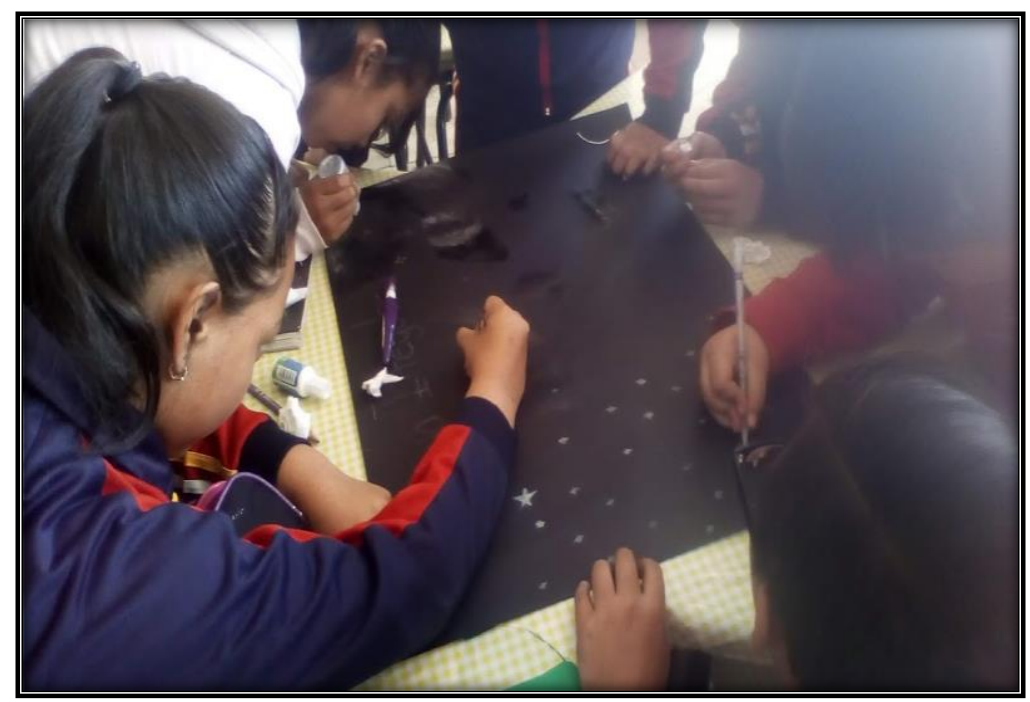

Figura 3. Actividades grupales, alumnos de $6^{\circ}$ "B" trabajando en equipo

Las actividades realizadas tienen como propósito generar ambientes de aprendizajes favorables en cada sesión ya que la sana convivencia favorece la creación de este tipo de ambientes, "Se denomina ambientes de aprendizaje al espacio donde se desarrolla la comunicación y las interacciones que posibilitan el aprendizaje” (SEP, 2011, p. 28)

El principio pedagógico número tres del mismo plan de estudios 2011 señala "generar ambientes de aprendizaje" razón por la cual las actividades del PISE se orientaron. En la asignatura de español se realizaron versos para un amigo, los alumnos tenían que escribir un verso desde los valores dirigido hacia una persona seleccionada aleatoriamente, haciendo uso de elementos poéticos como rima, metáfora, símil y reiteración.

La actividad calidad de vida se aplicó el día 5 de junio del Día Mundial del medio ambiente. Fue una actividad que favorecía a la convivencia escolar desde el sustento teórico de Geografía y Formación Cívica y Ética, la actividad consistió en investigar, diseñar y presentar un cartel sobre el cuidado del medio ambiente y el cuidado de sí mismo, a partir de esto, se interactuó con docentes en formación, docentes titulares y con compañeros alumnos, del eje de intersección disciplinar para favorecer la reflexión y la convivencia como un punto influyente sobre la calidad de vida de los alumnos. 
"Se trata de dar a los estudiantes una oportunidad de descubrir sus propias verdades y sus propias maneras de cambiar las condiciones sociales y económicas vigentes. Se trata de hacer de la educación una experiencia fascinante y capacitadora, más que una tarea penosa que debe realizarse con unos principios formulados desde el exterior" (Unwin, 1992, p. 45).

\section{Etapa 5: Evaluación y resultados}

La evaluación del PISE y las actividades del proyecto didáctico se realizó mediante encuestas aplicadas a los docentes titulares y alumnos; la encuesta para los docentes, consta de diez preguntas y un apartado de sugerencias aplicadas a la docente titular de $6^{\circ}$ "B", el cuestionario aplicado a los alumnos (Figura 4), constan de cinco preguntas y un apartado de comentarios, estás fueron contestadas por alumnos del mismo grado.

Los docentes titulares consideran la intervención como favorable, bajo la creación de ambientes de aprendizaje sanos para una convivencia armónica entre los alumnos. Se planteó que las actividades eran acordes al PISE, generaron y promovieron una convivencia de forma pacífica y permitió la integración entre los alumnos.

Por otro lado, la encuesta aplicada a los alumnos arroja los siguientes resultados:

- El 98\% de los alumnos están de acuerdo con el tema que articuló las actividades y que pusieron en práctica los valores aprendidos.

- $95 \%$ de los alumnos consideran que las actividades les ayudaron a convivir con todos sus compañeros.

- $\quad 77 \%$ de los alumnos consideran que les fue de agrado realizar actividades con otros grupos.

- $78 \%$ de los alumnos consideran agradable el material empleado

- $\quad 93 \%$ de los alumnos consideran que aprendieron cosas nuevas. 


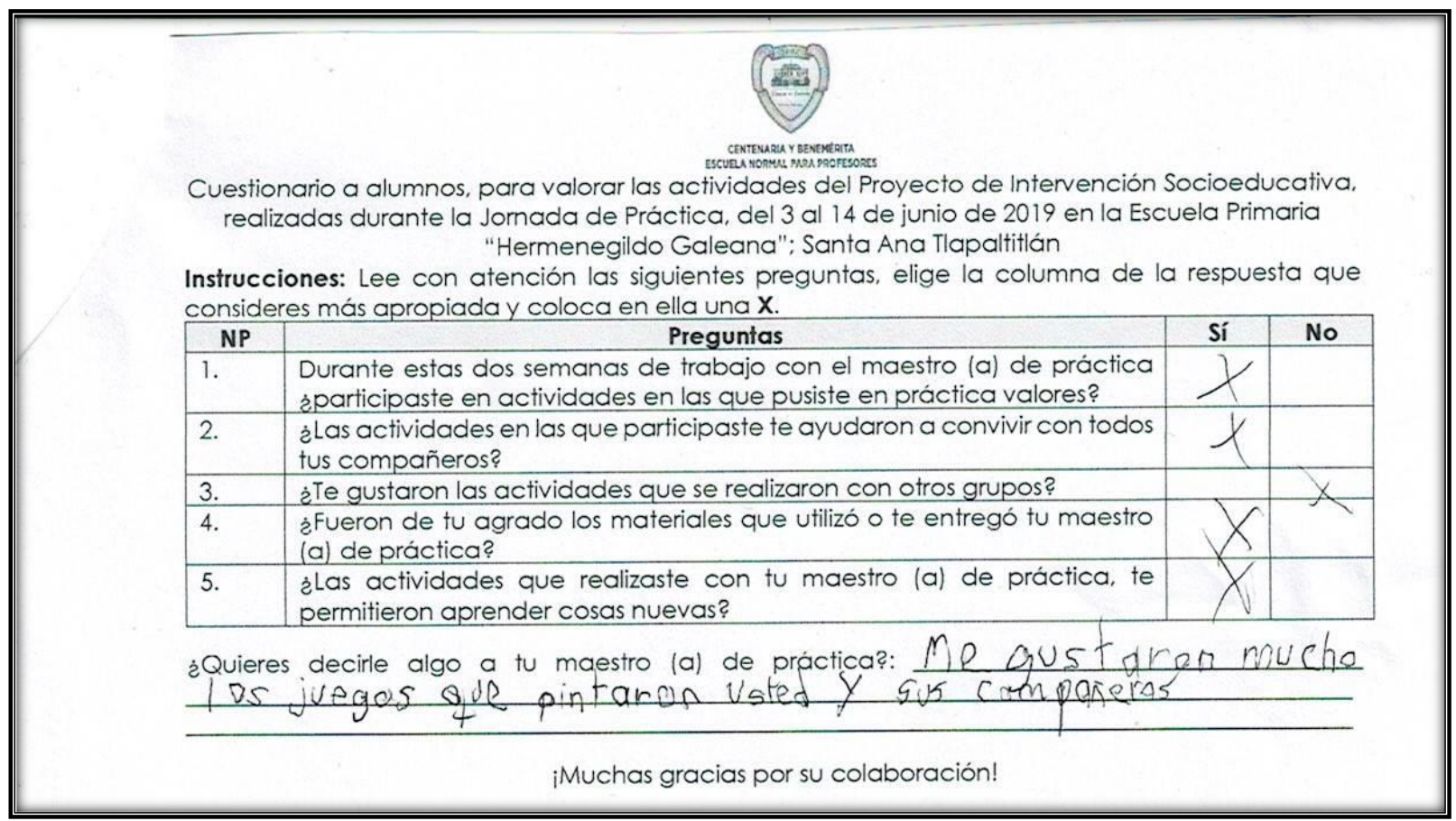

Figura 4. Cuestionario aplicado a los alumnos de sexto grado.

\section{Conclusiones}

La implementación de proyectos permite al docente conocer y aplicar críticamente los planes de estudios, al tener que relacionar las asignaturas que se imparten; si bien todo va relacionado, no se debe perder el enfoque de la enseñanza de cada asignatura. El proceso a través del cual se ha desarrollado la profesión docente; la influencia del contexto histórico y social, los principios filosóficos y valores en los que se sustenta mediante el análisis y debates de diferentes autores vistos.

El curso Diagnóstico e Intervención Socioeducativa, se aprendió a diseñar proyectos de trabajo para vincular las necesidades del entorno y la institución, con base en un diagnóstico a través de los instrumentos de observación para la realización de éste, su planificación e implementación, viéndose reflejado en las actividades implementadas.

El curso Formación Cívica y Ética se aprendió a diseñar ambientes de aprendizaje basados en diversas formas de interrelación que favorezcan la puesta en práctica de actitudes de convivencia con otro; el proyecto giró en torno a los valores y la convivencia. También, se revisó la actuación oportuna para prevenir y resolver situaciones de conflicto en la escuela utilizando 
herramientas de diálogo y negociación, favoreciendo la observación de las normas y un clima de respeto y empatía, pues era la base del PISE.

En Educación Geográfica se profundizó en mayor manera en los componentes del espacio geográfico (naturales, sociales, económicos, políticos, culturales y lingüísticos); un tema puede ser enseñado de manera global para que los alumnos aprecien cómo se relacionan diversos factores. También se profundizó en la comprensión de las características (lugar, medio, región, territorio y paisaje) y las categorías (localización, distribución, diversidad, temporalidad y la relación antecedente consecuente).

La Educación Artística, se adquirieron las competencias para gestionar recursos materiales, financieros, humanos que apoyan actividades que fomenten el contacto de los alumnos con el arte y desarrollen su capacidad de apreciación artística. Al mismo tiempo a través de la apreciación y expresión de las artes por medio de la obra de teatro, se valoró la riqueza de las manifestaciones culturales contribuyendo a su preservación y cuidado.

El curso Producción de Textos Académicos se aprendió a aplicar sistemáticamente las etapas del proceso de escritura de textos académicos, así como las estrategias discursivas y las herramientas metodológicas de cada tipo de documento, a través de la investigación realizada en el curso y la aplicación en la primaria, la cual se refleja en la planeación didáctica. En el curso de inglés B1, se adquirió el lenguaje básico y necesario para llevar a la escuela primaria actividades y dinámicas en inglés, desarrollando la adquisición de un segundo idioma a los alumnos la jornada de práctica.

Por último, en Proyectos de Intervención Socioeducativa se aprendió a elaborar proyectos que articulan diversos campos disciplinares para desarrollar un conocimiento integrado en los alumnos. El proyecto que se realizó tomando en cuenta una problemática con la colaboración de todos los cursos de la academia del sexto semestre, y con todas las disciplinas de la primaria.

Se emplearon los recursos y medios didácticos idóneos para la generación de aprendizajes de acuerdo con los niveles de desempeño esperados en el grado escolar, pues se 
diseñaron materiales innovadores y fuera de la cotidiano para la práctica, a la vez de que se llevó un proyecto didáctico transdisciplinar que colaboró con el PISE.

Se promovieron actividades que favorecen la equidad de género, tolerancia y respeto, contribuyendo al desarrollo personal y social de los alumnos, esta competencia se adquirió durante todo el proceso del proyecto que giró alrededor de la temática de la convivencia, los alumnos aprendieron a convivir tomando como base fundamental la aplicación de los valores y principios que incluyen la participación de todos, sin exclusiones ni distinciones. Fueron, capaces de comprender que la convivencia es fundamental para elevar la calidad de vida de las personas, y que toda persona necesita estar bien consigo mismo para poder apoyar a los demás y que los otros los apoyen a ellos.

Los métodos globalizadores, son una oportunidad de aprendizaje para que los estudiantes tengan acceso a una mayor educación integral, se favorece el desarrollo de competencias de diferentes asignaturas al mismo tiempo, por lo que resulta efectivo en cuestiones de optimización de tiempo y recursos porque se ocuparon los mismos materiales para diferentes lecciones.

La mayor parte de las actividades del PISE tuvieron un gran éxito, permitieron favorecer la convivencia escolar. El PISE muestra la importancia de la intervención educativa, permite transformar la realidad, para mejorar se debe crear instituciones educativas capaces de enseñar para la vida, desde base en los valores, principios cívicos, y derechos humanos.

\section{Referencias bibliográficas}

Aguilar, E. A. (1998). Cómo elaborar un proyecto: guía para diseñar proyectos sociales y culturales (14 ed.). Buenos Aires, Argentina: Lumen/Humanistas.

Alsina, P. (2012). 7 ideas clave: la competencia cultural y artística. Barcelona: GRAÓ.

Arias, F. (1999). El proyecto de investigación. Guía para su elaboración (3a ed.). Caracas: Orial.

Barriga, F. D. (2006). La conducción de la enseñanza mediante proyectos situados. En F. D. Barriga, Enseñanza situada (págs. 29-51). Ciudad de México: McGrawl-Hill. 
Gómez, L. F. (2003). La educación: entre la transmisión y el cambio. Sinéctica, 19-25.

Kant, I. (1781). Crítica de la razón pura. Prusia.

Risco, E. (2010). Orientaciones metodológicas para la investigación-acción. Perú: Biblioteca Nacional de Perú .

Rojas, A. L. (2014). Aportes de la sociología al estudio de la educación (Autores clásicos). Revista educación, 33-58.

SEP . (2017). Aprendizajes clave para la educación integral. Ciudad de México.

SEP. (2011). Principios pedagógicos . En SEP, Plan de estudios 2011 (pág. 28). Ciudad de México.

SEP. (2011). Programa de estudios 6o. Ciudad de México.

SEP. (2013). Guía para la elaboración de proyectos didácticos. Ciudad de México.

Unwin, T. (1994). El lugar de la geografía. Madrid: Editorial Cátedra . 